\title{
White Matter Alterations in the Brains of Patients with Active, Remitted, and Cured Cushing Syndrome: A DTI Study
}

P. Pires, A. Santos, Y. Vives-Gilabert, S.M. Webb, A. Sainz-Ruiz, E. Resmini, I. Crespo, M. de Juan-Delago, and B. Gómez-Anson $O-\equiv \triangle D_{2}$

\begin{abstract}
BACKGROUND AND PURPOSE: Cushing syndrome appears after chronic exposure to elevated glucocorticoid levels. Cortisol excess may alter white matter microstructure. Our purpose was to study WM changes in patients with Cushing syndrome compared with controls by using DTI and the influence of hypercortisolism.
\end{abstract}

MATERIALS AND METHODS: Thirty-five patients with Cushing syndrome and 35 healthy controls, matched for age, education, and sex, were analyzed through DTI (tract-based spatial statistics) for fractional anisotropy, mean diffusivity, axial diffusivity, and radial diffusivity (general linear model, family-wise error, and threshold-free cluster enhancement corrections, $P<.05$ ). Furthermore, the influence of hypercortisolism on WM DTI changes was studied by comparing 4 subgroups: 8 patients with Cushing syndrome with active hypercortisolism, 7 with Cushing syndrome with medication-remitted cortisol, 20 surgically cured, and 35 controls. Cardiovascular risk factors were used as covariates. In addition, correlations were analyzed among DTI values, concomitant 24-hour urinary free cortisol levels, and disease duration.

RESULTS: There were widespread alterations (reduced fractional anisotropy, and increased mean diffusivity, axial diffusivity, and radial diffusivity values; $P<.05$ ) in patients with Cushing syndrome compared with controls, independent of the cardiovascular risk factors present. Both active and cured Cushing syndrome subgroups showed similar changes compared with controls. Patients with medically remitted Cushing syndrome also had reduced fractional anisotropy and increased mean diffusivity and radial diffusivity values, compared with controls. No correlations were found between DTI maps and 24-hour urinary free cortisol levels or with disease duration.

CONCLUSIONS: Diffuse WM alterations in patients with Cushing syndrome suggest underlying loss of WM integrity and demyelination. Once present, they seem to be independent of concomitant hypercortisolism, persisting after remission/cure.

ABBREVIATIONS: $A D=$ axial diffusivity; $C S=$ Cushing syndrome; $F A=$ fractional anisotropy; $M D=$ mean diffusivity; $R D=$ radial diffusivity; $\mathrm{UFC}=$ urinary free cortisol

C ushing syndrome (CS) is a rare endocrine disease with a prevalence of approximately 40 cases per million inhabitants, ${ }^{1}$ in which diagnosis and, therefore, treatment are often delayed for months or years. It is caused by excessive cortisol exposure due to an adrenocorticotropin-secreting pituitary adenoma (Cushing disease), an adrenal adenoma, or an ectopic secretion of adreno-

Received October 11, 2014; accepted after revision December 14.

From the Asociación para la Innovación en Análisis, Gestión y Procesamiento de Datos Científicos y Tecnológicos (P.P., Y.V.-G.), Barcelona, Spain; Departments of Endocrinology/Medicine (P.P., A.S., S.M.W., E.R., I.C.), Hospital Sant Pau, IIB-Sant Pau, Instituto de Salud Carlos III, and Universitat Autònoma de Barcelona, Barcelona, Spain; Port d'Informació Científica (Y.V.-G., A.S.-R.), Bellaterra, Barcelona, Spain; Institut de Fisica d'Altes Energies (A.S.-R.), Barcelona, Spain; and Neuroradiology Unit (M.d.J.-D., B.G.-A.), Hospital Sant Pau, IIB-Sant Pau, Universitat Autònoma de Barcelona, Barcelona, Spain.

This work was supported by grants from the Instituto de Salud Carlos III, Spanish Ministry of Science and Innovation (MICINN, FIS080302 and FIS 070770), and the European Commission (ERCUSYN PHP800200).

corticotropin by tumors in other organs. ${ }^{2}$ It causes a range of physical and psychological symptoms, including depression and apathy. ${ }^{3}$ The so-called glucocorticoid cascade hypothesis, based on animal models, associates the harmful effects of glucocorticoid excess on the structure, function, and vitality of brain cells. ${ }^{4}$ Excessive exposure to glucocorticoid leads to changes in plasticity, reduced neurogenesis, and cell loss in the hippocampus. ${ }^{5}$ Besides, cortisol induces myelin loss around axons in postnatal rats, ${ }^{6}$ and more recent evidence indicates that prolonged exposure to ele-

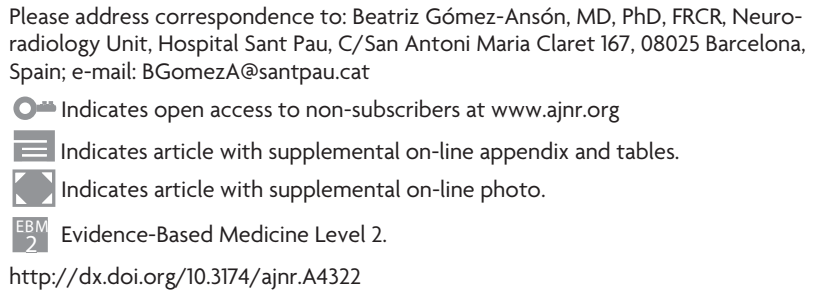
radiology Unit, Hospital Sant Pau, C/San Antoni Maria Claret 167, 08025 Barcelona, Spain; e-mail: BGomezA@santpau.cat

-- Indicates open access to non-subscribers at www.ajnr.org

$\equiv$ Indicates article with supplemental on-line appendix and tables.

Indicates article with supplemental on-line photo.

EBM Evidence-Based Medicine Level 2.

http://dx.doi.org/10.3174/ajnr.A4322 
Demographic and clinical characteristics of healthy controls and patients with active, remitted, and cured CS $^{\mathrm{a}}$

\begin{tabular}{|c|c|c|c|c|c|c|}
\hline & $\begin{array}{l}\text { Healthy } \\
\text { Controls }\end{array}$ & $\begin{array}{l}\text { Patients with } \\
\text { Active CS }\end{array}$ & $\begin{array}{l}\text { Patients with } \\
\text { Remitted CS }\end{array}$ & $\begin{array}{l}\text { Patients with } \\
\text { Cured CS }\end{array}$ & $F / \chi^{2}$ & $P$ Value \\
\hline Age (yr) & $42.3 \pm 10.4$ & $41.9 \pm 8.7$ & $47.1 \pm 10.0$ & $41.4 \pm 10.0$ & 5.9 & NS \\
\hline $\operatorname{Sex}(M / F)$ & $6: 29$ & $1: 7$ & $1: 6$ & $4: 16$ & 0.3 & NS \\
\hline Years of education & $13.5 \pm 3.4$ & $14.4 \pm 3.0$ & $12.9 \pm 2.3$ & $13.4 \pm 3.1$ & 0.3 & NS \\
\hline 24-Hour urinary free cortisol & $133 \pm 52$ & $543 \pm 307$ & $152 \pm 74$ & $122 \pm 75$ & 26.3 & $<.001^{\mathrm{b}}$ \\
\hline Smoking status (nonsmoker/smoker/ex-smoker) & $12 / 12 / 11$ & $7 / 1 / 0$ & $2 / 4 / 1$ & $9 / 5 / 6$ & 10.4 & NS \\
\hline Disease duration (mo) & NA & $77.6 \pm 77.4$ & $44.6 \pm 21.9$ & $64.6 \pm 34.0$ & 0.99 & NS \\
\hline Origin (pituitary/adrenal/ectopic/AIMAH) & NA & $5 / 3 / 0 / 0$ & $3 / 2 / 1 / 1$ & $17 / 2 / 0 / 0$ & 10.8 & NS \\
\hline Hypertension (no/yes) & $35 / 0$ & $4 / 4$ & $3 / 4$ & $13 / 7$ & 20.9 & $<.001^{\mathrm{c}}$ \\
\hline Hypercholesterolemia (no/yes) & $31 / 4$ & $7 / 1$ & $7 / 0$ & $17 / 3$ & 1.2 & NS \\
\hline Hypertriglyceridemia (no/yes) & $29 / 6$ & $6 / 2$ & $3 / 4$ & $9 / 11$ & 10.5 & $.015^{\mathrm{d}}$ \\
\hline Central obesity (no/yes) & $25 / 10$ & $2 / 6$ & $0 / 7$ & $9 / 11$ & 15.6 & $.001^{\mathrm{e}}$ \\
\hline Body mass index ${ }^{\mathrm{g}}$ (no/overweight/obesity) & $22 / 11 / 2$ & $2 / 5 / 1$ & $1 / 3 / 3$ & $8 / 9 / 3$ & 12.6 & $.049^{f}$ \\
\hline
\end{tabular}

Note:-NS indicates not significant; AIMAH, ACTH-independent macronodular adrenal hyperplasia.

a Data are expressed as mean $\pm \mathrm{SD}$.

${ }^{b}$ Healthy controls vs active CS $(<.001)$; active CS vs remitted CS $(<.001)$; active CS vs cured CS $(<.001)$.

${ }^{c}$ Healthy controls vs active CS $(<.001)$; healthy controls vs remitted CS $(<.001)$; healthy controls vs cured CS $(<.001)$.

${ }^{\mathrm{d}}$ Healthy controls vs remitted CS (.043); healthy controls vs cured CS (.006).

e Healthy controls vs active CS (.015); healthy controls vs remitted CS (.001).

${ }^{f}$ Healthy controls vs remitted CS (.015).

Body mass index: normal, $\leq 25$; overweight, 25-30; obesity, $>30$.

vated glucocorticoid may alter oligodendrocyte-mediated remyelination. ${ }^{7,8}$ Thus, cortisol excess most likely alters the microstructure of cerebral white matter, targeting the myelinated axonal tracts in the brains of patients with CS. Moreover, hypercortisolism frequently determines diabetes mellitus, central obesity, and thrombophilia, which increase cardiovascular disease, ${ }^{9}$ the main cause of mortality in CS, even after biochemical cure. ${ }^{10}$ These comorbidities may also affect cerebral WM microstructure in CS. ${ }^{11}$

A few imaging studies have investigated brain structures in patients with CS. ${ }^{12,13}$ Starkman et al, ${ }^{14}$ using MR imaging, found a relationship among hippocampal volume reduction, memory dysfunction, and elevated cortisol levels in CS. More recent studies, including CT and MR imaging, have described loss of brain volume in CS. ${ }^{15}$ With MR imaging and automated volumetry, hippocampal volume reductions were found in patients with CS with severe memory deficits. ${ }^{16}$ A more recent study investigated WM with DTI in patients in long-term remission of Cushing disease and observed widespread abnormalities. The authors also observed a loss of integrity of the uncinate fasciculus, which was related to the severity of depression. ${ }^{17}$ They concluded that structural changes in WM integrity in the brain persisted after hypercortisolism cure $^{17}$; but patients with active disease were not included, and cardiovascular risk factors were not reported.

DTI is an MR imaging technique that allows noninvasive, in vivo study of the brain by assessing the motion of water molecules along and across neural axons. ${ }^{18,19}$ Different DTI maps show the WM architecture ${ }^{20}$ and can depict microstructural WM abnormalities. Fractional anisotropy (FA) reflects WM integrity, while increases in mean diffusivity (MD) may be caused by demyelination or edema. ${ }^{21}$ Decreases in axial diffusivity (AD) indicate axonal loss, ${ }^{22}$ while increased radial diffusivity (RD) is related to demyelination. ${ }^{21}$

The aim of this study was to investigate cerebral WM microstructure in patients with CS compared with healthy controls by using DTI. It was hypothesized that WM integrity in the brain of patients with CS was altered, reflecting microstructural changes, and that these alterations persisted after remission or cure of hypercortisolism.
A first DTI analysis was performed comparing patients with CS with healthy controls, followed by a second analysis in which patients were separated into active CS, remitted CS, and cured CS. Comorbidities known to increase cardiovascular risk were investigated as covariates. Correlations of WM changes with 24-hour urinary free cortisol (UFC) values and the duration of hypercortisolism were also investigated.

\section{MATERIALS AND METHODS \\ Participants}

Thirty-five patients with CS and 35 healthy control volunteers matched for sex, age ( \pm 3 years), and years of education $( \pm 3$ years) were included. Eight patients had active hypercortisolism, 7 had remitted hypercortisolism on medical therapy, and 20 were cured after surgery (Table). Remission of hypercortisolism was considered when patients achieved adrenal insufficiency or morning cortisol suppression $(<50 \mathrm{nmol} / \mathrm{L},<1.8 / \mu \mathrm{g} / \mathrm{dL})$ after 1 mg dexamethasone overnight and had repeatedly normal 24 -hour UFC levels ( $<280 \mathrm{nmol} / 24$ hours). Disease duration, 24-hour UFC levels, and clinical variables related to cardiovascular risk (smoking, hypertension, dyslipidemia, central obesity, and increased body mass index) were collected (Table). Disease duration was defined by the endocrinologist in charge as the time from symptom onset until remission of hypercortisolism after treatment. At diagnosis, duration of hypercortisolism was estimated by personal interview and detailed review of medical records and photographs of patients. All information was written or kept in clinical records, together with biochemical data. Patients with diabetes mellitus were excluded because it is known to affect WM. ${ }^{23}$

All subjects were right-handed and signed an informed consent after approval of the study by the ethics committee of the hospital. No subject had brain injury or clinical cerebrovascular disease.

\section{Image Processing}

DTI processing was performed at the computing of Port d'Informació Científica in Barcelona through a designed, portalbased platform, including fMRI of the Brain Software Library 
(FSL; Version 4.1.4; http://www.fmrib.ox.ac.uk/fsl). ${ }^{24}$ Images were first corrected for head motion and eddy currents by an affine registration. The first $3 \mathrm{D}$ volume was extracted as the image reference by using the Brain Extraction Tool (http://fsl.fmrib.ox. ac.uk/fsl/fslwiki/BET). ${ }^{25}$ Voxelwise images of FA, MD, AD, and $\mathrm{RD}$ maps were generated by using the $\mathrm{fMRI}$ of the Brain Diffusion Toolbox (http://www.fmrib.ox.ac.uk/fsl/fdt/index.html) by fitting diffusion tensors at each voxel. Next, the tract-based spatial statistics package (http://fsl.fmrib.ox.ac.uk/fsl/fslwiki/TBSS) ${ }^{26}$ was applied for a voxelwise statistical analysis. First, the fMRI of the Brain Linear Image Registration Tool (FLIRT; http://www. fmrib.ox.ac.uk/) was used to align all FA images into a standard space (fMRI of the Brain 58-FA). Then, a mean skeleton of all FA images was built by selecting the main WM tracts, thinned with a 0.2 threshold value (excluding all FA voxels of $<0.2$ ) to increase consistency. MD, AD, and RD maps followed the same TractBased Spatial Statistics procedure of nonlinear warps and skeleton projection to obtain estimated vectors for each.

MR imaging acquisition and statistical analysis are detailed in the On-line Appendix.

\section{RESULTS}

\section{Analysis 1: Comparison between Patients with CS and Controls}

Demographic and Clinical Variables. Because of the matched study design, no differences in age, sex, or years of education between patients with CS and controls were present. Differences were found for comorbidities: hypertension (0 controls/15 patients with CS; $P<.001$ ), hypertriglyceridemia (6 controls/17 patients with CS; $P=.004)$, central obesity (10 controls/24 patients with CS; $P<.001$ ), and body mass index (22, healthy, $<25$; 11 overweight, between 25 and 30; and 2 obese, $>30$ controls versus 11 healthy, 17 overweight, and 7 obese patients with CS; $P=.019)$. To correct for these differences, we included these comorbidities as covariates in the DTI analysis. No differences in smoking ( 12 nonsmokers, 12 smokers, and 11 ex-smokers among controls versus 18 nonsmokers, 10 smokers, and 7 ex-smokers in patients with CS) or in the prevalence of hypercholesterolemia (4 healthy controls versus 4 patients with CS) were observed between groups.

\section{DTI Analysis}

The Figure shows widespread FA decreases $(A)$ and MD (B), AD $(C)$, and $\mathrm{RD}(D)$ increases in all patients with CS compared with controls $(P<.05)$. No differences were found in the reverse comparisons (ie, controls had neither lower FAs nor higher MD, AD, or RDs than patients with CS).

\section{Analysis 2: Comparison between Patients with Active, Remitted, and Cured CS and Controls}

Demographic and Clinical Characteristics. No differences were found in age, sex, or years of education between controls and any of the CS subgroups. Twenty-four-hour UFC levels in patients with active CS were higher than in those in the other 3 groups. The prevalence of hypertension, hypertriglyceridemia, central obesity, and increased body mass index differed among groups, so they were analyzed as covariates in DTI analysis (Table). Smoking and hypercholesterolemia did not differ among groups. No differences in disease duration or etiology among the CS groups were found.

\section{DTI Analysis}

On-line Fig 1 shows voxels/regions having decreased FA $(A)$ and increased $\mathrm{MD}(B), \mathrm{AD}(C)$, and $\mathrm{RD}(D)$ values in patients with active CS compared with controls $(P<.05)$. No differences were found in the reverse contrasts (ie, controls had neither lower FA nor higher $\mathrm{MD}, \mathrm{AD}$, or $\mathrm{RD}$ values than patients with active $\mathrm{CS}$ ).

On-line Fig 2 shows voxels/regions having decreased FA $(A)$ and increased $\mathrm{MD}(B)$ and $\mathrm{RD}(C)$ values $(P<.05)$ in patients with remitted CS compared with controls. No differences were found in the reverse contrasts (ie, controls had neither lower FA nor higher $\mathrm{MD}$ or $\mathrm{RD}$ values than patients with active $\mathrm{CS}$ ). No differences in $\mathrm{AD}$ values among groups were seen.

On-line Fig 3 shows voxels/regions having decreased FA $(A)$ and higher $\mathrm{MD}(B), \mathrm{AD}(C)$, and $\mathrm{RD}(D)$ values $(P<.05)$ in patients with cured CS compared with controls. No differences were found in the reverse contrasts (ie, controls had neither lower FA nor higher $\mathrm{MD}, \mathrm{AD}$, or $\mathrm{RD}$ values than patients with cured CS).

No differences were found in any DTI maps/values (FA, MD, $\mathrm{AD}$, or $\mathrm{RD}$ ) among the 3 subgroups of patients with CS (active, remitted, and cured).

\section{Correlation Analysis}

No correlations were found for any DTI maps (FA, MD, AD, and $\mathrm{RD})$ with the 24-hour UFC levels of healthy controls $(n=28)$ or patients with CS $(n=34)$ or with disease duration in patients with CS.

\section{DISCUSSION}

This study demonstrates widespread WM alterations on DTI in the brains of patients with CS compared with healthy controls, which persist after controlling for cardiovascular risk factors (hypertension, dyslipidemia, and obesity). The pattern of WM involvement, with decreased FA and increased $\mathrm{MD}, \mathrm{AD}$, and $\mathrm{RD}$, suggests an underlying loss of WM integrity and predominant demyelination. The subgroup analysis demonstrates this same pattern of WM involvement in patients with active, remitted, and cured CS, without any correlation among DTI values, concomitant 24-hour UFC levels, or disease duration. These results imply that WM damage does not depend on current hypercortisolism because it persists after remission/cure of CS. These findings support the recent concept that once WM damage has occurred after early exposure to hypercortisolism, it is not completely reversible, despite successful treatment with surgery or medical therapy. It adds to the growing body of evidence that residual morbidity persists after endocrine control of CS. ${ }^{17}$

Although most DTI studies refer to FA values, the other DTI maps/parameters provide additional information on WM microstructure that expands the understanding of WM integrity. Reduced FA values indicate loss of fiber coherence and therefore of WM integrity. An increase in overall water diffusion in all directions (MD) may reflect demyelination ${ }^{27}$ or edema ${ }^{21}$; more specif- 


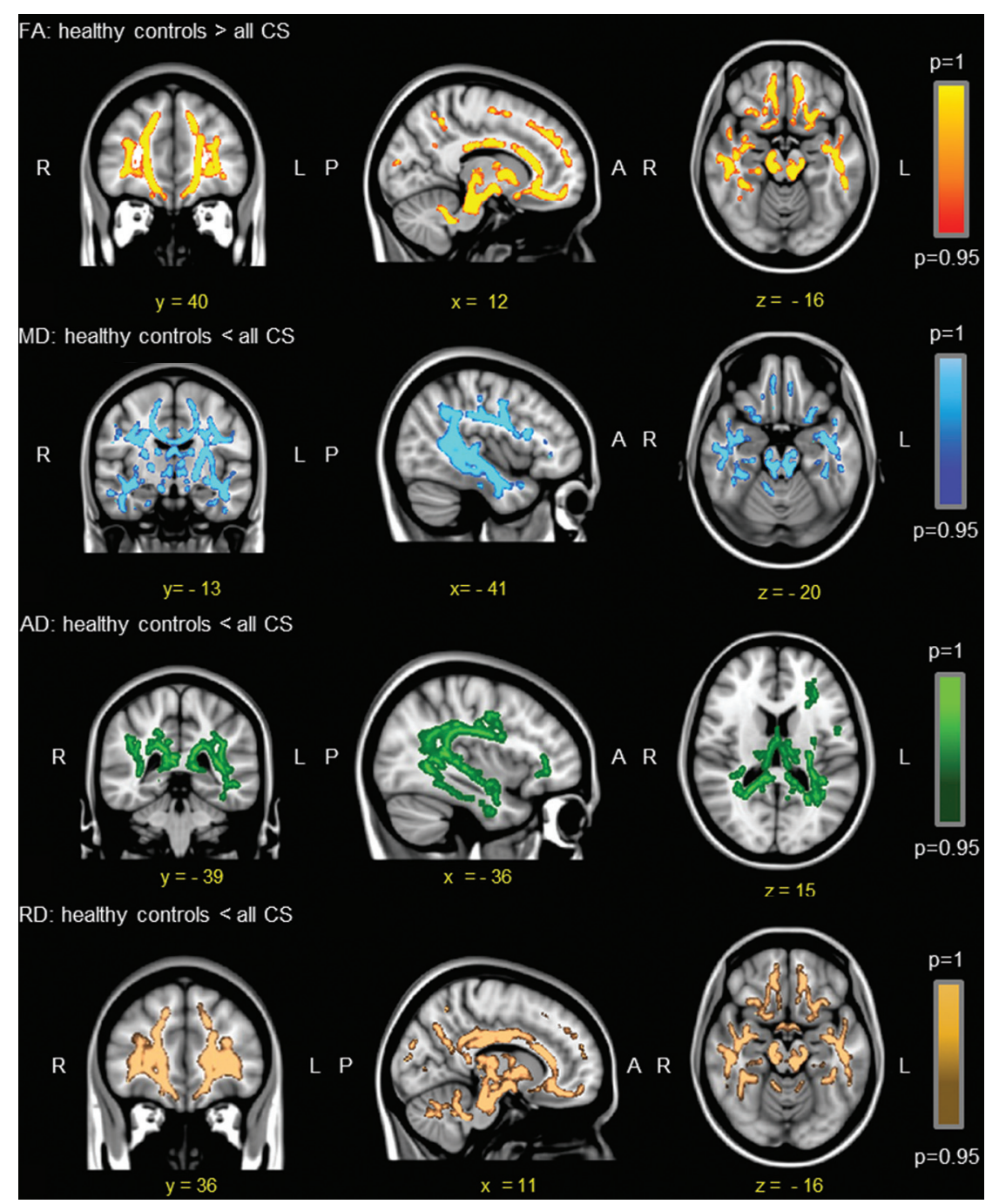

FIGURE. Differences $(P<.05)$ in DTI maps among all patients with CS and controls by using hypertension, hypertriglyceridemia, central obesity, and body mass index as covariates.
They also found increased RD values, similar to our results, indicating predominant underlying demyelination in altered WM tracts. The observed increases in RD should, nevertheless, be interpreted with caution, especially in regions containing white matter tract crossings, as has been previously reported. ${ }^{31}$ Our results expand the findings not only to patients in long-term remission but also in those with active hypercortisolism and those with remitted CS on medical therapy. Furthermore, cardiovascular risk factors, which may also damage the brain WM, were controlled for in our study. Evidence indicates that glucocorticoid may contribute to brain aging ${ }^{5}$ and produce WM changes on $\mathrm{DTI}^{32}$ similar to those found in CS. The pattern of WM involvement in aging has been termed "chronic WM degeneration" and includes increased extracellular volume (edema) and lower membrane attenuation as a consequence of myelin loss, smaller axonal ratio volume, higher axonal interspace, and reductions in extracellular tortuosity. ${ }^{33}$ These microstructural WM changes are similar to the DTI changes observed in patients with CS in our study.

Some studies have reported a certain degree of reversibility of brain ${ }^{34}$ and hippocampal volume $\operatorname{loss}^{35}$ after hypercortisolism correction. However, metabolic abnormalities in the hippocampi of patients with cured CS, namely per-
ction/loss and glial proliferation, have resistent neuronal dysfunction/loss and glial proliferation, have re-
cently been observed, ${ }^{36}$ which agree with the DTI alterations we have now observed in patients with remitted and cured CS. Most interesting, it has been suggested that chronic exposure to glucocorticoid in the central nervous system could cause inflammation, ${ }^{37}$ which may trigger WM microstructure damage in patients with CS, as found in the present study. Moreover, high cortisol levels are known to increase the prevalence of cardiovascular risk factors ${ }^{9,11}$ such as hypertension, dyslipidemia, and obesity, which could also affect WM microstructure. Therefore, we corrected for these cardiovascular risk factors, which differed among groups of patients with CS; but WM damage was observed independent of the existence or absence of these comorbidities. We excluded patients and controls with diabetes, another disease known to damage brain WM. Our results lead us to hypothesize that exposure to hypercortisolism plays the major role, possibly mediated by inflammation, ${ }^{37}$ and is responsible for the persistence of WM damage in remitted/cured patients with $\mathrm{CS}^{38}$

Our study has some limitations: first, the small sample of subjects included, which is difficult to overcome in a rare endocrine 
disease like CS. Compared with controls, patients with remitted CS showed decreased FA and increased MD and RD only, with no changes in $\mathrm{AD}$, these results being different from those observed in patients with active and cured CS; this lack of change in AD may be due to the small sample size. Second, the DTI study, although methodologically robust, is cross-sectional and includes patients with CS in different stages of the disease (active, remitted, and cured). To clearly define the influence of hypercortisolism on WM alterations, larger longitudinal studies are desirable. Finally, our study did not address the functional significance of WM alterations and whether they relate to psychology.

\section{CONCLUSIONS}

WM damage on DTI in the brains of patients with CS suggests widespread integrity loss and predominant demyelination. These alterations seem to be independent of concomitant hypercortisolism and the presence or absence of other cardiovascular risk factors like hypertension, dyslipidemia, and obesity, and they persist despite remission/cure. When they occur and the mechanisms and the potential modifiers involved remain unclear.

\section{ACKNOWLEDGMENTS}

We thank patients and healthy volunteers who participated in the current study for their kind cooperation.

Disclosures: Patricia Pires—RELATED: Grant: Spanish Ministry of Science and Innovation (FIS080302, FIS 070770).* Alicia Santos—RELATED: Grant: Ministerio de Ciencia y educación, ${ }^{\star}$ Comments: Public Spanish grant FIS080302. Susan M. WebbRELATED: Grant: Instituto de Salud Carlos III, Ministry of Economy and Competitivity, and Ministry of Health, Spain, ${ }^{\star}$ Comments: This was a public competitive grant for which I am the principal investigator. Eugenia Resmini-RELATED: Grant: Ministerio de Ciencia e Innovacion (FIS 080302).* Iris Crespo-RELATED: Grant: FIS 080302 "Neuroradiological, Neuropsychological and Clinical Study in Endogenous Hypercortisolism: Comparison between Cushing's Syndrome and Chronic Major Depressive Disorder"; funding agency: Instituto de Salud Carlos III; duration of the project: from 2009 to 2011, Principal Investigator: Susan M. Webb. *Money paid to the institution.

\section{REFERENCES}

1. Newell-Price J. Cushing's syndrome. Clin Med 2008;8:204-08

2. Hatipoglu BA. Cushing's syndrome. J Surg Oncol 2012;106: 565-71

3. Guaraldi F, Salvatori R. Cushing syndrome: maybe not so uncommon of an endocrine disease. J Am Board Fam Med 2012;25: 199-208

4. Sapolsky RM, Krey LC, McEwen BS. The neuroendocrinology of stress and aging: the glucocorticoid cascade hypothesis. Endocr Rev 1986;7:284-301

5. Goosens KA, Sapolsky RM. Stress and glucocorticoid contributions to normal and pathological aging. In Riddle DR, ed. Brain Aging: Models, Methods, and Mechanisms. Boca Raton: CRC Press; 2007:305-22

6. Bohn MC, Friedrich VL Jr. Recovery of myelination in rat optic nerve after developmental retardation by cortisol. J Neurosci 1982;2:1292-98

7. Alonso G. Prolonged corticosterone treatment of adult rats inhibits the proliferation of oligodendrocyte progenitors present throughout white and gray matter regions of the brain. Glia 2000;31:219-31

8. Miyata S, Koyama Y, Takemoto K, et al. Plasma corticosterone activates SGK1 and induces morphological changes in oligodendrocytes in corpus callosum. PLoS One 2011;6:e19859
9. Fallo F, Sonino N. Should we evaluate for cardiovascular disease in patients with Cushing's syndrome? Clin Endocrinol 2009;71: $768-71$

10. Aulinas A, Valassi E, Webb SM. Prognosis of patients treated for Cushing syndrome. Endocrinol Nutr 2014;61:52-61

11. Miljic P, Miljic D, Cain JW, et al. Pathogenesis of vascular complications in Cushing's syndrome. Hormones (Athens) 2012;11: 21-30

12. Trethowan WH, Cobb S. Neuropsychiatric aspects of Cushing's syndrome. AMA Arch Neurol Psychiatry 1952;67:283-309

13. Cope O, Raker JW. Cushing's disease: the surgical experience in the care of 46 cases. $N$ Engl J Med 1955;253:119-27

14. Starkman MN, Gebarski SS, Berent S, et al. Hippocampal formation volume, memory dysfunction, and cortisol levels in patients with Cushing's syndrome. Biol Psychiatry 1992;32:756-65

15. Simmons NE, Do HM, Lipper MH, et al. Cerebral atrophy in Cushing's disease. Surg Neurol 2000;53:72-76

16. Resmini E, Santos A, Gómez-Anson B, et al. Verbal and visual memory performance and hippocampal volumes, measured by 3-Tesla magnetic resonance imaging, in patients with Cushing's syndrome. J Clin Endocrinol Metab 2012;97:663-71

17. van der Werff SJ, Andela CD, Nienke Pannekoek J, et al. Widespread reductions of white matter integrity in patients with long-term remissions of Cushing's disease. Neuroimage Clin 2014;4:659-67

18. Johansen-Berg H, Rushworth MF. Using diffusion imaging to study human connectional anatomy. Annu Rev Neurosci 2009;32: 75-94

19. Le Bihan D, Breton E, Lallemand D, et al. MR imaging of intravoxel incoherent motions: application to diffusion and perfusion in neurologic disorders. Radiology 1986;161:401-07

20. Le Bihan D. Looking into the functional architecture of the brain with diffusion MRI. Nat Rev Neurosci 2003;4:469-80

21. Alexander AL, Lee JE, Lazar M, et al. Diffusion tensor imaging of the brain. Neurotherapeutics 2007;4:316-29

22. Budde MD, Xie M, Cross AH, et al. Axial diffusivity is the primary correlate of axonal injury in the experimental autoimmune encephalomyelitis spinal cord: a quantitative pixelwise analysis. J Neurosci 2009;29:2805-13

23. Hsu JL, Chen YL, Leu JG, et al. Microstructural white matter abnormalities in type $\mathbf{2}$ diabetes mellitus: a diffusion tensor imaging study. Neuroimage 2012;59:1098-105

24. Smith SM, Jenkinson M, Woolrich MW, et al. Advances in functional and structural MR image analysis and implementation as FSL. Neuroimage 2004;23(suppl 1):S208-19

25. Smith SM. Fast robust automated brain extraction. Hum Brain Mapp 2002;17:143-55

26. Smith SM, Jenkinson M, Johansen-Berg H, et al. Tract-based spatial statistics: voxelwise analysis of multi-subject diffusion data. Neuroimage 2006;31:1487-505

27. Horsfield MA, Jones DK. Applications of diffusion-weighted and diffusion tensor MRI to white matter diseases: a review. NMR Biomed 2002;15:570-77

28. Beaulieu $C$. The basis of anisotropic water diffusion in the nervous system: a technical review. NMR Biomed 2002;15:435-55

29. Song SK, Yoshino J, Le TQ, et al. Demyelination increases radial diffusivity in corpus callosum of mouse brain. Neuroimage 2005;26:132-40

30. Wheeler-Kingshott CA, Cercignani M. About "axial” and "radial" diffusivities. Magn Res Med 2009;61:1255-60

31. Jones DK, Knösche TR, Turner R. White matter integrity, fiber count, and other fallacies: the do's and don'ts of diffusion MRI. Neuroimage 2013;73:239-54

32. Minati L, Grisoli M, Bruzzone MG. MR spectroscopy, functional MRI, and diffusion-tensor imaging in the aging brain: a conceptual review. J Geriatr Psychiatry Neurol 2007;20:3-21

33. Burzynska AZ, Preuschhof C, Bäckman L, et al. Age-related differences in white matter microstructure: region-specific patterns of diffusivity. Neuroimage 2010;49:2104-12 
34. Bourdeau I, Bard C, Noël B, et al. Loss of brain volume in endogenous Cushing's syndrome and its reversibility after correction of hypercortisolism. J Clin Endocrinol Metab 2002;87:1949-54

35. Starkman MN, Giordani B, Gebarski SS, et al. Decrease in cortisol reverses human hippocampal atrophy following treatment of Cushing's disease. Biol Psychiatry 1999;46:1595-602

36. Resmini E, Santos A, Gómez-Anson B, et al. Hippocampal dysfunc- tion in cured Cushing's syndrome patients, detected by (1) H-MRspectroscopy. Clin Endocrinol (Oxf) 2013;79:700-07

37. Sorrells, Sapolsky RM. An inflammatory review of glucocorticoid actions in the CNS. Brain Behav Immun 2007;21:259-72

38. Barahona MJ, Sucunza N, Resmini E, et al. Persistent body fat mass and inflammatory marker increases after long-term cure of Cushing's syndrome. J Clin Endocrinol Metab 2009;94:3365-71 